\title{
Influence of Controlled Breathing on Cerebrovascular Control During Upright Tilt
}
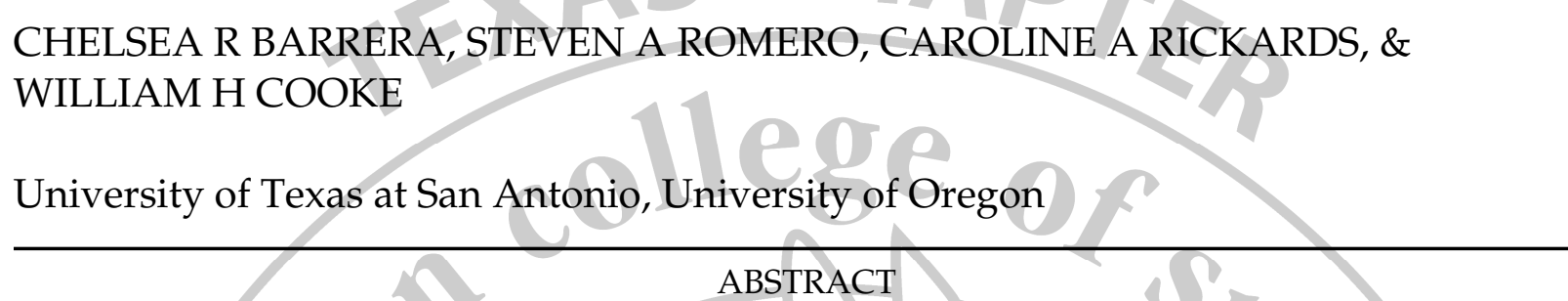

Arterial pressures oscillate with the frequency of respiration, and these oscillations are translated directly to the cerebrovasculature. For this reason, intrinsic cerebrovascular control is assessed at the low frequency (LF; .07-.2 Hz). When humans breathe spontaneously, it is possible that breathing frequency encroaches on these non-respiratory rhythms, thereby confounding the interpretation of intrinsic cerebrovascular control. PURPOSE: To test the hypothesis that controlled breathing (CB) decreases, and spontaneous breathing (SB) increases the reliance of cerebral blood velocity on arterial pressure within the LF range in both the supine and upright postures. METHODS: We recorded ECG, finger arterial pressure (Finometer), transcranial Doppler ultrasound of the middle cerebral artery, and end-tidal $\mathrm{CO}_{2}$ in 20 healthy male volunteers $(24 \pm 2 \mathrm{yrs})$. Ten subjects breathed in time to a metronome set at a pace of 15 breaths/min (CB), and ten subjects breathed spontaneously (SB). Both groups were studied in the supine and head-up tilt (HUT) positions for 5-min. Reliance of mean cerebral blood velocity ( $\mathrm{CBV}_{\text {mean }}$ ) on mean arterial pressure (MAP) was assessed over the LF with cross-spectral coherence analysis $(\mathrm{COH})$. RESULTS: Respiratory rates were not different between CB and SB during supine $(p=.86)$, but were

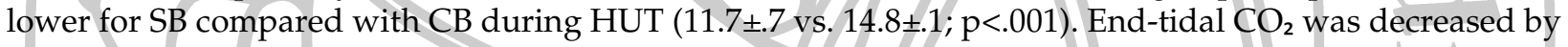
$\mathrm{CB}$ during both supine and $\mathrm{HUT}(\mathrm{p}<.05)$. $\mathrm{CBV}_{\text {mean }}$ was decreased with $\mathrm{CB}$ during supine $(\mathrm{p}=.04)$, but was similar between CB and SB during HUT $(p=14)$. Neither LFMAP nor LFCBV mean oscillations were different in the supine position ( $p>$.6), but were increased (with a trend for LFMAP) with SB during HUT $\left(\mathrm{p}=.003\right.$ for $\mathrm{LF} C B V_{\text {mean }}$ and $\mathrm{p}=.09$ for LFMAP). $\mathrm{COH}$ was not different in the supine position between CB and SB $(.42 \pm .05$ for $\mathrm{CB}$ and $.61 \pm .06 \mathrm{SB} ; \mathrm{p}=.13)$, but was lower for $\mathrm{CB}$ in the HUT position (.55 \pm .05 for $\mathrm{CB}$ and $.78 \pm .08$ for $\mathrm{SB} ; \mathrm{p}=.02$ ). CONCLUSION: Reliance of $\mathrm{CBV}_{\text {mean }}$ on MAP is increased in the LF range when subjects breathe spontaneously during HUT. We attribute changes in $\mathrm{COH}$ during HUT to entrainment of respiratory-mediated arterial pressure fluctuations on the cerebrovasculature. However, the potential confounding influence of hypocapnia warrants further investigation.

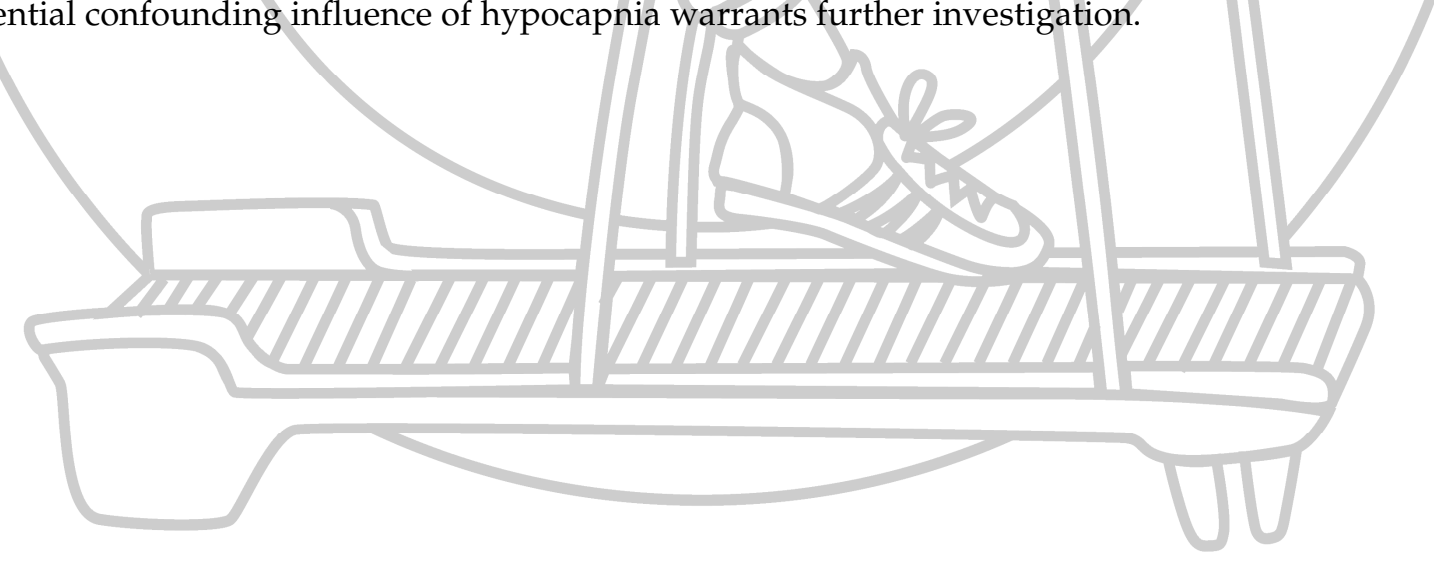

